\title{
Generalized Field Propagator for Electromagnetic Scattering and Light Confinement
}

\author{
Olivier J. F. Martin* \\ IBM Research Division, Zurich Research Laboratory, 8803 Rueschlikon, Switzerland \\ Christian Girard \\ Laboratoire de Physique Moléculaire UA CNRS 772, Université de Franche Comté, 2530 Besançon, France \\ Alain Dereux \\ Institute for Studies in Interface Sciences, Facultés Universitaires N.-D. de la Paix, 5000 Namur, Belgium
}

(Received 9 August 1994)

\begin{abstract}
We present a new theoretical and numerical framework for the study of the optical properties of micrometric and nanometric three-dimensional structures of arbitrary shape. We show that the field distribution induced inside and outside such a structure by different external monochromatic sources can be obtained from a unique generalized field propagator expressed in direct space. An application of the method to the confinement of optical fields due to the scattering of subwavelength objects is presented.
\end{abstract}

PACS numbers: 42.25.-p, 02.30.Tb, 02.60.Nm, 61.16.Ch

Over the past few years, considerable efforts have been devoted to the understanding of the response properties of micrometric and nanometric structures isolated in gas phase [1] or integrated on a surface [2]. Recent continuous progress in scanning near-field optical microscopy (SNOM) [3-6] has strongly enhanced our insight into the field distributions in the vicinity of such subwavelength structures. From a theoretical point of view, dealing with low-symmetry, three-dimensional (3D) systems renders the intricate problem related to the electromagnetic boundary conditions at the material interfaces intractable. Therefore, most of the numerical approaches based on the matching of these boundary conditions encounter difficulties when applied to geometrically complex mesoscopic and subwavelength systems made of realistic materials. This fact was demonstrated by the theoretical challenge raised by the development of SNOM.

In order to circumvent this obstacle inherent to the matching of electromagnetic boundary conditions, we present in this Letter a new theoretical framework for a large class of problems dealing with 3D objects of arbitrary shape and dielectric functions. More precisely, we show that the entire field distribution induced by different sources, inside and outside a 3D structure, can be derived from a unique generalized field propagator expressed in direct space. This approach is based on Green's dyadic technique. Although the power of this technique has long been recognized, its use was impeded by the difficult construction of Green's dyadics associated with complex geometries. In this Letter, we point out that electromagnetic scattering theory gains much efficiency by adopting certain procedures from quantum scattering theory. Particularly, the introduction of a dyadic Dyson's equation enables the straightforward construction of Green's dyadics associated with arbitrarily complex geometries.
Let us consider a nonmagnetic scattering system described by a dielectric tensor $\varepsilon(\mathbf{r}, \omega)=\varepsilon_{r}(\omega)+\varepsilon_{s}(\mathbf{r}, \omega)$, embedded in an infinite homogeneous reference medium $\varepsilon_{r}(\omega)$. This scattering system must not necessarily be a single region, but can be formed by disconnected parts embedded in the reference system, with the tensor $\varepsilon_{s}(\mathbf{r}, \omega)$ vanishing outside of the scattering system.

When an incident field $\mathbf{E}^{0}(\mathbf{r})$ (a monochromatic field with the usual $\exp [-i \omega t]$ time dependence is assumed throughout this Letter, but the method is able to handle arbitrary incident waves) impinges on that system, the scattered field $\mathbf{E}(\mathbf{r})$ is a solution of the vectorial wave equation

$$
-\nabla \times \nabla \times \mathbf{E}(\mathbf{r})+k^{2} \varepsilon_{r}(\omega) \mathbf{E}(\mathbf{r})+k^{2} \varepsilon_{s}(\mathbf{r}, \omega) \mathbf{E}(\mathbf{r})=\mathbf{0},
$$

where $k$ is the wave number in vacuum.

Introducing the operators $\mathbf{L}, \mathbf{e}_{r}$, and $\mathbf{e}_{s}$ for $-\nabla \times \nabla \times$, $k^{2} \varepsilon_{r}(\omega)$, and $k^{2} \varepsilon_{s}(\mathbf{r}, \omega)$, respectively, we can rewrite Eq. (1) in operator notation as

$$
\left(\mathbf{L}+\mathbf{e}_{r}+\mathbf{e}_{s}\right) \mathbf{E}=\mathbf{0} .
$$

The incident field $\mathbf{E}^{0}(\mathbf{r})$ verifies, in the same notation,

$$
\left(\mathbf{L}+\mathbf{e}_{r}\right) \mathbf{E}^{0}=\mathbf{0} .
$$

The field propagator or Green's tensor $\mathbf{G}\left(\mathbf{r}, \mathbf{r}^{\prime}, \omega\right)$ associated with a given physical system describes the response of this system to a pointlike source excitation. This dyadic tensor is closely related to the field susceptibility of the system [7] and has proven to be extremely useful in the context of surface science and quantum electrodynamics [8-10]. More recently, it has also been used to describe nonradiative energy transfer occurring in nearfield optics [11,12].

The Green's operator $\mathbf{G}^{0}$ associated with the reference system is defined by

$$
\left(\mathbf{L}+\mathbf{e}_{r}\right) \mathbf{G}^{0}=\mathbf{1},
$$


where $\mathbf{1}$ is the unit operator, whereas Green's operator $\mathbf{G}$ associated with the complete system is defined by

$$
\left(\mathbf{L}+\mathbf{e}_{r}+\mathbf{e}_{s}\right) \mathbf{G}=\mathbf{1} .
$$

Setting Eq. (2) equal to (3) and using Eq. (5), we obtain

$$
\mathbf{E}=\mathbf{E}^{0}-\mathbf{G e}_{s} \mathbf{E}^{0} \equiv \mathbf{K E}^{0} .
$$

Equation (6) defines the generalized propagator $\mathbf{K}=\mathbf{1}$ $\mathbf{G e}_{s}$. In the $\mathbf{r}$ representation, Eq. (6) becomes

$$
\begin{aligned}
\mathbf{E}(\mathbf{r}) & =\int d \mathbf{r}^{\prime}\left[\delta\left(\mathbf{r}-\mathbf{r}^{\prime}\right)-k^{2} \mathbf{G}\left(\mathbf{r}, \mathbf{r}^{\prime}, \omega\right) \cdot \varepsilon_{s}\left(\mathbf{r}^{\prime}, \omega\right)\right] \cdot \mathbf{E}^{0}\left(\mathbf{r}^{\prime}\right) \\
& \equiv \int d \mathbf{r}^{\prime} \mathbf{K}\left(\mathbf{r}, \mathbf{r}^{\prime}, \omega\right) \cdot \mathbf{E}^{0}\left(\mathbf{r}^{\prime}\right)
\end{aligned}
$$

The remarkable property of $\mathbf{K}\left(\mathbf{r}, \mathbf{r}^{\prime}, \omega\right)$ is that it depends only on the geometry of the scattering system; it does not depend on the incident field $\mathbf{E}^{0}(\mathbf{r})$. Therefore, this unique propagator contains the entire response of the scattering system at frequency $\omega$ and, once it has been computed, can be used with Eq. (7) to obtain the scattering field $\mathbf{E}(\mathbf{r})$ for an arbitrary incident field $\mathbf{E}^{0}(\mathbf{r})$.

Note that in a first stage one can restrain the calculation to the field $\mathbf{E}(\mathbf{r})$ inside the scattering system and therefore limit the integration in Eq. (7) to the scattering system. The field $\mathbf{E}(\mathbf{r})$ at any point of the reference system can then be obtained at a later stage using the Lippmann-Schwinger equation [13]

$$
\mathbf{E}(\mathbf{r})=\mathbf{E}^{0}(\mathbf{r})-k^{2} \int d \mathbf{r}^{\prime} \mathbf{G}^{0}\left(\mathbf{r}, \mathbf{r}^{\prime}, \omega\right) \varepsilon_{s}\left(\mathbf{r}^{\prime}, \omega\right) \cdot \mathbf{E}\left(\mathbf{r}^{\prime}\right) .
$$

Equation (8) is obtained from Eqs. (2) and (3); it shows that the knowledge of the field in the scattering system is sufficient to compute the response at any position of the reference system using Green's tensor $\mathbf{G}^{0}\left(\mathbf{r}, \mathbf{r}^{\prime}, \omega\right)$ associated with the homogeneous reference system. This tensor is known analytically for a homogeneous 3D system [14,15]:

$$
\begin{aligned}
\mathbf{G}^{0}\left(\mathbf{r}, \mathbf{r}^{\prime}, \omega\right)=- & \left(\mathbf{1}-\frac{1-i k_{r} R}{k_{r}^{2} R^{2}} \mathbf{1}\right. \\
& \left.-\frac{-3+3 i k_{r} R+k_{r}^{2} R^{2}}{k_{r}^{2} R^{4}} \mathbf{R} \mathbf{R}\right) \frac{\exp \left[i k_{r} R\right]}{4 \pi R},
\end{aligned}
$$

where $R=|\mathbf{R}|=\left|\mathbf{r}-\mathbf{r}^{\prime}\right|$ and $k_{r}^{2}=\varepsilon_{r}(\omega) k^{2}$. Let us note that one could also consider a planar surface as a reference system and, using the corresponding Green's tensor $\mathbf{G}_{\text {surface }}^{0}\left(\mathbf{r}, \mathbf{r}^{\prime}, \omega\right)$, obtain the generalized field propagator for a scattering system lying on that surface.

To compute Green's tensor G required to obtain the generalized propagator $\mathbf{K}$, we use Dyson's equation [13]

$$
\mathbf{G}=\mathbf{G}^{0}-\mathbf{G}^{0} \mathbf{e}_{s} \mathbf{G} .
$$

Equation (10) is easily derived from Eqs. (4) and (5). Instead of inverting Eq. (10) with a standard procedure, we use the following original iterative scheme.
Let us consider the discretized Dyson's equation

$$
\mathbf{G}_{i, j}=\mathbf{G}_{i, j}^{0}-k^{2} \sum_{p=1}^{N} \mathbf{G}_{i, p}^{0} \varepsilon_{p}^{s} \cdot \mathbf{G}_{p, j} \Delta_{p},
$$

where the scattering system has been divided into $N$ meshes of respective size $\Delta_{i}$ centered at $\mathbf{r}_{i}, i=1, \ldots, N$. The discretized variables $\mathbf{G}_{i, j}$ and $\varepsilon_{i}^{s}$ have been introduced for $\mathbf{G}\left(\mathbf{r}_{i}, \mathbf{r}_{j}, \omega\right)$ and $\varepsilon_{s}\left(\mathbf{r}_{i}, \omega\right)$, respectively. Instead of solving Eq. (11) directly, we construct the discretized propagator $\mathbf{G}_{i, j}$ iteratively by considering each mesh of the discretized scattering system successively. For example, at step $m$ of the calculation, we compute the propagator $\mathbf{G}_{i, j}^{m}$ associated with mesh $m$. Equation (11) becomes

$$
\mathbf{G}_{i, j}^{m}=\mathbf{G}_{i, j}^{m-1}-k^{2} \mathbf{G}_{i, m}^{m-1} \boldsymbol{\varepsilon}_{m}^{s} \cdot \mathbf{G}_{m, j}^{m} \Delta_{m} .
$$

The operator $\mathbf{G}_{i, j}^{m-1}$ is used instead of $\mathbf{G}_{i, j}^{0}$ in Eq. (12) to take into account the $m-1$ previous meshes of the scattering system. The solution of Eq. (12) is extremely simple. Starting with $i=m$, we obtain $\mathbf{G}_{m, j}^{m}$ by solving a small $(3 \times 3)$ system of equations. The value of $\mathbf{G}_{m, j}^{m}$ is then used to compute $\mathbf{G}_{i, j}^{m}$ for $i \neq m$ using Eq. (12). This procedure, repeated over every mesh of the system, leads to the propagator $\mathbf{G}_{i, j}^{N} \equiv \mathbf{G}_{i, j}$ associated with the entire system. This numerical scheme is extremely stable compared, for example, to the matching methods, and can therefore be used to obtain $\mathbf{G}$ for a very large physical system [16].

Particular attention must be devoted to the divergence of $\mathbf{G}_{i, j}^{0}$ for $i=j$. This divergence requires the appropriate renormalization procedure, as described in detail by Yaghjian in a different context [17]. These diagonal elements of $\mathbf{G}_{i, j}^{0}$ play an extremely important role because they account for the depolarization of the matter induced by the applied field. Indeed, the response of dielectric matter to an external field $\mathbf{E}^{0}(\mathbf{r})$ results in a depolarization field such that the total field (external + depolarization) fulfills the boundary conditions at the interfaces between different materials [18]. In this way, the intricate electromagnetic boundary conditions are automatically satisfied for any kind of material and object shape.

To assess the power and versatility of the generalized propagator and corresponding numerical scheme, we investigate the field distribution in the vicinity of subwavelength dielectric systems. This problem is particularly relevant in the context of SNOM, where the imaging properties of an object are determined by the field spawned at its vicinity.

Let us consider as a scattering system a 3D letter "F" with a dielectric constant $\varepsilon_{\mathrm{s}}=2.25$ embedded in vacuum $\left(\varepsilon_{\mathrm{r}}=1\right)$. As this shape has a very low symmetry, different incident fields should lead to completely different responses of the system. The size of the letter is $30 \mathrm{~nm} \times 50 \mathrm{~nm}$, and its thickness $7.5 \mathrm{~nm}$; it is discretized 
with three layers of 136 meshes each. The mesh size is $\Delta_{x}=\Delta_{y}=\Delta_{z}=2.5 \mathrm{~nm}$. The 3D letter is illuminated from its side by a plane wave of wavelength $633 \mathrm{~nm}$ and unit amplitude.

The generalized propagator is used to compute the field inside the object, and Eq. (8) is then applied to obtain the field in an observation plane parallel to the letter face, placed a distance $d$ below the letter. This observation plane, located in the reference system, is introduced merely for the convenience of data visualization. Indeed the field can be computed for any position of the system using Eq. (8).

In Fig. 1 we present contour plots of the field amplitude in the vicinity of the scattering object $(d=5 \mathrm{~nm})$; the spacing between the contour lines always represents $10 \%$ of the corresponding amplitude range. The projection of the scattering system on the observation plane is also shown. Two different polarizations are investigated: incident field parallel to the observation plane (transverse electric field, TE) and incident field orthogonal to the observation plane (transverse magnetic field, TM).

In the TE case, strong field gradients appear along the object sides that are orthogonal to the incident electric
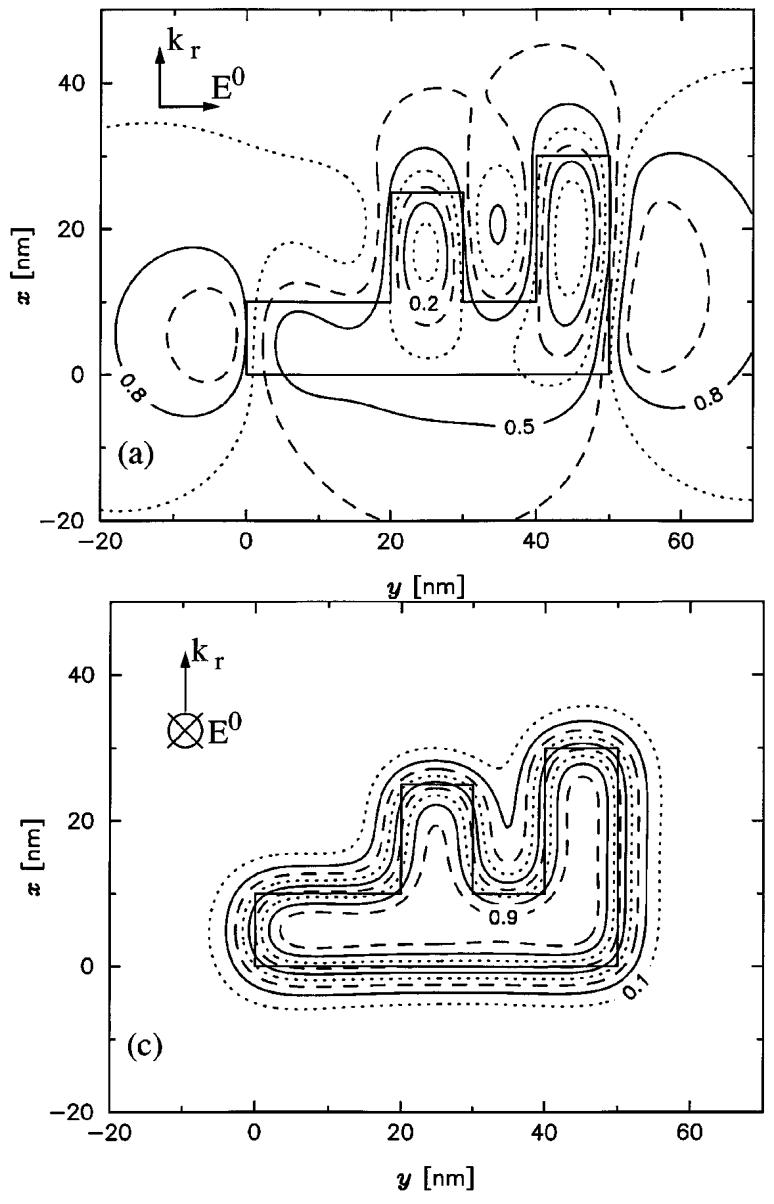

field [Figs. 1(a) and 1(b)]. Thus different sides of the object are enhanced, depending on the orientation of the incident electric field, and the field pattern strongly depends on the propagation direction of the incident field. The field intensity in the detection plane is highest immediately outside the object and decreases below it, leading to an inverse contrast image of the object. This phenomenon is related to depolarization effects enhanced by the small size of the object. Indeed, when the size of the object is larger than the wavelength, these depolarization effects are hidden by the complicated field pattern that can arise in the structure.

For TM fields, the behavior is completely different. Now the isoamplitude lines perfectly follow the contour of the object [Figs. 1(c) and 1(d)]. Furthermore, the image does not depend on the orientation of the incident field, and the field pattern always reproduces the object shape. A strong confinement of the field below the object is also visible. The electric field is now mainly polarized along the $z$ direction, and the depolarization in the object occurs in that direction as well. Therefore, the various depolarization effects that occur in the $z$ direction cannot be resolved by scanning in a plane orthogonal to that
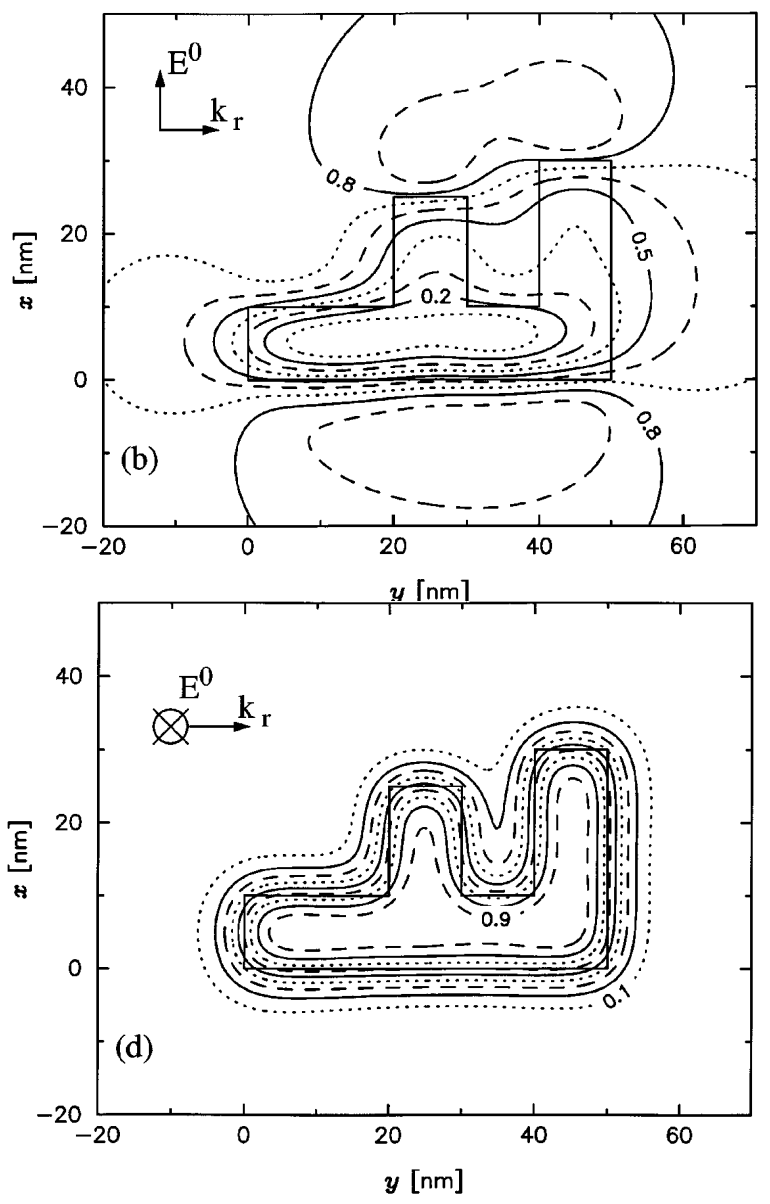

FIG. 1. Field amplitude in an observation plane located $d=5 \mathrm{~nm}$ below the scattering system. (a),(b) TE incident field and (c),(d) TM incident field. Two different propagation directions are investigated for each polarization. The orientation $\mathbf{E}^{0}$ of the incident field and its propagation direction $\mathbf{k}_{\mathrm{r}}$ are represented in each figure. 


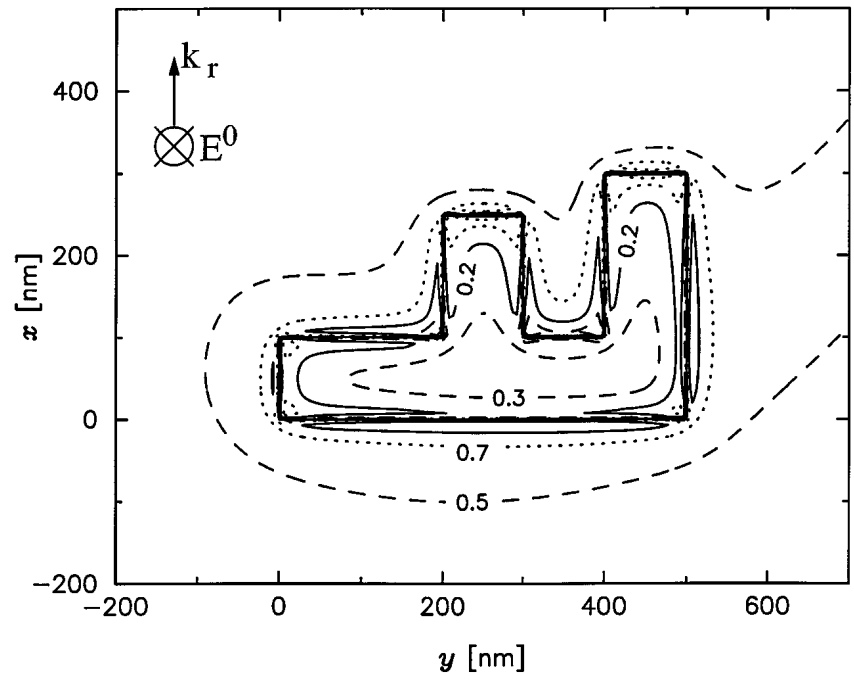

FIG. 2. Same situation as in Fig. 1(c), but now the lateral dimensions of the letter are 10 times larger.

direction, and only the overall response over the entire system thickness is measured. Such a confined field can be recorded by an SNOM probing device, which explains how SNOM can reach a resolution far beyond the diffraction limit. Therefore, whereas a TE illumination emphasizes the sides of the object, a TM illumination leads to strongly confined fields that reproduce the entire object shape.

When the observation distance increases, the field behavior changes, and the field pattern becomes similar to that scattered by a point dipole. Both polarizations now produce somewhat similar diffraction patterns, but for TM field the maximum field amplitude corresponds to the position of the scattering system, whereas for TE polarization this maximum appears ahead of the object in the propagation direction. Therefore, TM illumination is best suited for the localization of objects in SNOM. Contrary to the near field, the field at larger distances depends on the propagation direction of the incident field for both polarizations.

Let us emphasize that all the results presented in Fig. 1 were obtained with the same generalized propagator $\mathbf{K}\left(\mathbf{r}, \mathbf{r}^{\prime}, \omega\right)$. The response of the system to the different incident fields was then computed using Eq. (7). The calculation of the generalized propagator for the system under study took $5.5 \mathrm{~min}$ on an IBM RISC system/6000 model 970 workstation. The generation of the field in the scattering system using Eq. (7) and the calculation of the field in the observation plane (with 6300 discretization points) using Eq. (8) took 2 and 41 s, respectively.

When the size of the scattering system increases, the field confinement observed for a small system disappears. This is visible in Fig. 2, where we report the field ampli- tude at the same observation distance from an object of the same thickness and dielectric function as that in Fig. 1, but 10 times larger; the incident field is TM polarized. Now the field is no longer confined in the object, and the maximum field amplitude is obtained outside the object. As the object is now larger than the effective wavelength, a more complicated field structure can be excited inside the latter. However, strong field gradients perfectly follow the entire object outline. For TE illumination, the behavior is similar to that of a small object, and strong field gradients appear along the object sides that are orthogonal to the incident electric field.

The generalized propagator presented in this Letter should be useful in numerous domains of electromagnetics where the response of geometrically complex systems to arbitrary excitations is required.

*Present address: Institute for Field Theory and High Frequency Electronics, Swiss Federal Institute of Technology, ETH-Zentrum, 8092 Zurich, Switzerland.

[1] C. F. Bohren and D. R. Huffman, Absorption and Scattering of Light by Small Particles (Wiley, New York, 1983).

[2] See, for example, the special issue of Phys. Today 46, No. 6 (1993), and references therein.

[3] D. W. Pohl, W. Denk, and M. Lanz, Appl. Phys. Lett. 44, 651 (1984).

[4] R. C. Reddick, R. J. Warmack, and T.L. Ferrell, Phys. Rev. B 39, 767 (1989).

[5] D. Courjon, K. Sarayeddine, and M. Spajer, Opt. Commun. 71, 23 (1989).

[6] E. Betzig and R. J. Chichester, Science 262, 1422 (1993).

[7] G. S. Agarwal, Phys. Rev. A 11, 230 (1975).

[8] F. Toigo, A. Marvin, V. Celli, and N. R. Hill, Phys. Rev. B 15, 5618 (1977).

[9] A. A. Maradudin and W. Zierau, Phys. Rev. B 14, 484 (1976).

[10] H. Metiu and P. Das, Annu. Rev. Phys. Chem. 35, 507 (1984).

[11] A. Dereux and D. W. Pohl, in Near Field Optics, edited by D.W. Pohl and D. Courjon, NATO ASI Series E, Vol. 242 (Kluwer, Dordrecht, 1993), p. 189.

[12] C. Girard and A. Dereux, Phys. Rev. B 49, 11344 (1994).

[13] E. N. Economou, Green's Functions in Quantum Physics (Springer, Berlin, 1990), 2nd ed.

[14] H. Levine and J. Schwinger, Commun. Pure Appl. Math. 3, 355 (1950).

[15] P.M. Morse and H. Feshbach, Methods of Theoretical Physics (McGraw-Hill, New York, 1953).

[16] O. J.F. Martin, A. Dereux, and C. Girard, J. Opt. Soc. Am. A 11, 1073 (1994).

[17] A. D. Yaghjian, Proc. IEEE 68, 248 (1980).

[18] C. Kittel, Introduction to Solid State Physics (Wiley, New York, 1986), 6th ed. 